\title{
ङु \\ Spectral bandwidth reduction of Thomson scattered light by pulse chirping
}

\author{
Isaac Ghebregziabher, B. A. Shadwick, and Donald Umstadter \\ Department of Physics and Astronomy, University of Nebraska-Lincoln, Lincoln, Nebraska 68588, USA
}

(Received 2 July 2012; published 19 March 2013)

\begin{abstract}
Based on single particle tracking in the framework of classical Thomson scattering with incoherent superposition, we developed a relativistic, three-dimensional numerical model that calculates and quantifies the characteristics of emitted radiation when a relativistic electron beam interacts with an intense laser pulse. This model has been benchmarked against analytical expressions, based on the plane wave approximation to the laser field, derived by Esarey et al. [Phys. Rev. E 48, 3003 (1993)]. For laser pulses of sufficient duration, we find that the scattered radiation spectrum is broadened due to interferences arising from the pulsed nature of the laser. We find that by appropriately chirping the scattering laser pulse, spectral broadening can be minimized, and the peak on-axis brightness of the emitted radiation is increased by a factor of approximately 5 .
\end{abstract}

DOI: 10.1103/PhysRevSTAB.16.030705

PACS numbers: 52.38.Ph, 41.60.Ap

\section{INTRODUCTION}

Intense, tunable, ultrashort, collimated, polarized, and quasimonoenergetic radiation in the $\mathrm{x}$-ray and $\gamma$-ray region of the electromagnetic spectrum has potential applications in broad disciplines that extend to, but are not limited to, natural sciences and health sciences. Such high-energy radiation sources may be generated when a relativistic electron beam interacts with an intense laser pulse [1-3]. During the interaction, relativistic electrons oscillate in the electromagnetic field of the laser and emit radiation. The emission of electromagnetic radiation by the accelerated charges, also known as Thomson scattering, could be interpreted as scattering of the incident laser pulse by the relativistic electron beam. Thomson scattering is a classical description of the scattering process [4] and is valid as long as the energy of the scattered photon is much less than the electron energy, $\hbar \omega_{\mathrm{sc}} \ll \gamma m c^{2}$, where $\hbar$ is the reduced Planck's constant, $\gamma$ is the relativistic Lorentz factor of the electron beam, $m$ is the rest mass of an electron, $c$ is the speed of light in vacuum, and $\omega_{\mathrm{sc}}$ is the angular frequency of the scattered photon. This is the low energy classical limit of the general quantum mechanical inverse Compton scattering process. The scattered photon energies reported in this paper are much less than the energy of the electron and, hence, the terms Thomson scattering and inverse Compton scattering are used interchangeably.

The oscillation of an electron in an electromagnetic field is generally classified as linear or nonlinear, depending on the strength of the laser field. A useful parameter for determining the onset of a nonlinear regime is the dimensionless laser strength parameter, $a_{0}=q A / m c \approx$

Published by the American Physical Society under the terms of the Creative Commons Attribution 3.0 License. Further distribution of this work must maintain attribution to the author(s) and the published article's title, journal citation, and DOI.
$8.5 \times 10^{-10} \lambda_{0}[\mu \mathrm{m}] I^{1 / 2}\left[\mathrm{~W} / \mathrm{cm}^{2}\right]$, where $A$ is the magnitude of the laser vector potential, $I$ is the laser intensity, and $\lambda_{0}$ is the wavelength of the laser pulse. For $a_{0} \ll 1$, the electron oscillation is linear and thus the corresponding scattering is called linear Thomson scattering. In nonlinear Thomson scattering, $a_{0}>1$, the electron can absorb multiple photons simultaneously and emit a single photon of higher energy [5-7]. In addition to the onset of nonlinearity based on the strength of the laser pulse, signatures of nonlinearity in the scattered radiation spectrum can occur for arbitrarily low strength and sufficiently long laser pulses [8-11]. The origin of these nonlinear signatures due to the pulsed nature of the laser [12], as well as a method of minimizing them, is discussed in this paper. By considering laser intensities corresponding to $a_{0}<1$, the onset of nonlinearity due to the strength of the laser pulse is decoupled from that which is due to the finite temporal duration of the laser.

The general properties of Thomson scattered photons by relativistic electron beams, which have been studied extensively $[1,13]$, may be understood through Doppler effect considerations. A relativistically moving electron beam experiences the compressed wave front of the laser pulse. The maximum compression of the wave front occurs along the direction of the electron beam. The electron beam then scatters the compressed wave front and an observer in the far field detects angle-dependent high-energy photons. The detected photon energy is highest for a detector placed in the direction of the electron beam. In a counterpropagating interaction geometry (i.e. laser and electron beam collide head-on), the photon energy of the laser light is Doppler up-shifted by a factor of $4 \gamma^{2}$. For example, if $\gamma=500$, and the photon energy of the laser pulse is $1.5 \mathrm{eV}$, then the onaxis scattered photon energy is $1.5 \mathrm{MeV}$, corresponding to a million-fold increase in photon energy. The emitted radiation is well collimated, near monochromatic, and has a high degree of polarization [14], unlike that from 
bremsstrahlung radiation source [15-18], which is typically unpolarized and with a broad energy spectrum.

The generation of bright, picosecond-duration $\mathrm{x}$ rays and $\gamma$ rays through inverse Compton scattering has already been demonstrated with head-on collisions of intense laser pulses synchronized to picosecond-duration, high-energy electron beams generated with conventional radio frequency (rf)-based accelerators $[19,20]$. Ultrashort x rays, with moderate brightness, have also been demonstrated from the inverse Compton scattering of femtosecond lasers in a $90^{\circ}$ scattering geometry with a synchronized, rfaccelerated, high-energy electron beam [21]. In addition to the experimental demonstration of $\mathrm{x}$-ray and $\gamma$-ray sources, the backscattered radiation was used as a diagnostic tool for the electron beam [22-24], to generate polarized positrons [25] from dense targets, and to demonstrate nuclear fluorescence (proving the utility of the source for discerning isotope specific elements) [26-28].

The theory of Thomson backscattering of an infinitely long electromagnetic field by a relativistic electron beam has been well documented [1,2,29]. Moreover, extensive numerical studies have also been performed for scattering from a pulsed plane wave electromagnetic field. We extend these previous works by including the realistic sixdimensional nature of the electron beam as well as the three-dimensional nature of the focused electromagnetic pulse with curved wave fronts [30,31]. This treatment includes spectral broadening of the scattered light due to wave front curvature and finite temporal duration of the scattering laser, and broadening associated with the transverse and longitudinal emittances of the electron beam. Such a detailed calculation of Thomson scattering is necessary to provide not only a framework for the analysis of experimental results, but also a guide to the design of Thomson scattered x-ray sources.

In this paper, we discuss a numerical model that calculates the scattered radiation during the interaction of an intense laser pulse with an electron beam. In addition to benchmarking the code against previously reported results, we use it to demonstrate a technique to reduce the spectral bandwidth of Thomson scattered light by means of chirping the incident scattering laser pulse. Section II discusses the core ingredients of the model and Sec. III contains a comparison of the numerically calculated and analytically obtained radiation energy. Section IV discusses the effect of finite temporal width of the laser on the scattered spectrum as well as a method to overcome broadening due to the pulsed nature of the scattering laser. Section V provides a summary of results.

\section{MODELING}

The three-dimensional and relativistic Thomson code [32] is divided as follows: (1) six-dimensional phase space sampling of the relativistic electron beam, (2) classical electron dynamics for an electron in phase space, and
(3) calculation of the radiation across the electron beam phase space and three-dimensional laser focus. A linearly polarized laser pulse with a central wavelength $\lambda_{0}=800 \mathrm{~nm}$ is used for the results presented in this paper. The polarization and propagation directions of the laser are parallel to the $x$ and $z$ axis, respectively.

There have been other numerical models that are based on the cross section approach $[33,34]$. In such models the total number of scattered photons is obtained by the overlap integral of the product of the total Thomson cross section and the flux of incident laser photons in the rest frame of the electron beam. Such cross section-based models do not account for the spectral oscillations in the radiated spectrum due to the pulsed nature of the scattering laser [12]. Instantaneous electron dynamics in the laser pulse must be included to account for the oscillations in the radiated spectrum. In our model, electron dynamics are obtained by solving relativistic equations of motion; hence, our model is able to account for finite beam shape effects.

\section{A. Phase space sampling of the electron beam}

The electron beam, described by its average energy $E_{0}$, relative energy spread $\Delta E / E_{0}$, and divergence angle $\theta_{e}$, is sampled by a six-dimensional phase space distribution. The phase space coordinate, $\left(x, y, z, p_{x}, p_{y}, p_{z}\right)$, of each electron in the beam is generated with a normal random number distribution of particles with respective standard deviations $\Delta p_{x}=\theta_{e} \times p_{z 0}, \Delta p_{y}=\theta_{e} \times p_{z 0}, \Delta x=\sigma_{x e}$, $\Delta y=\sigma_{y e}$, and $\Delta z=c \times \tau_{e} / 2 \sqrt{2 \ln (2)}$, where $\tau_{e}$ is the full width at half maximum (FWHM) temporal duration of the electron beam. The longitudinal momentum spread is then obtained with $\Delta p_{z}=E\left(\Delta E / p_{z} c^{2}\right)-$ $p_{x}\left(\Delta p_{x} / p_{z}\right)-p_{y}\left(\Delta p_{y} / p_{z}\right)$. Since the electron beams considered here are relativistic with small divergence angles, the longitudinal momentum spread, $\Delta p_{z}$, of the electron beam is approximately equal to $\Delta E / c$.

\section{B. Accurate treatment of the laser field}

It is clear that the plane wave approximation to a laser field may be valid when the electron beam probes only the central region of the laser focus. In reality the laser focus has curved wave fronts that depend on space. When the beam waist of the scattering laser focus is comparable to the size of the electron beam, the plane wave approximation may not be valid. To leading order, the paraxial approximation for fields in the laser focus may be adopted to calculate the scattered radiation more accurately. In the paraxial approximation the laser electromagnetic fields are assumed to be transverse. The longitudinal field components, which become significant for a tightly focused laser beam, are neglected since the expansion parameter $\epsilon=\lambda / \pi s_{0}$ for a Gaussian beam, is very small for the laser focus beam waist radius $s_{0}$ used in this paper. The electric and magnetic field components of a linearly polarized 
laser pulse propagating along the $z$ axis under the paraxial approximation are $E_{x}=\tilde{E} f(\eta) \cos (\Phi+\alpha)$ and $B_{y}=\tilde{E} f(\eta) \cos (\Phi+\alpha) / c$, respectively, where $\tilde{E}=$ $E_{0}\left[s_{0} / s(z)\right] \exp \left[-r^{2} / s(z)^{2}\right], r^{2}=x^{2}+y^{2}, \Phi=\omega_{0} t-k z-$ $z r^{2} / z_{R} s(z)^{2}, \alpha=\arctan \left(z / z_{R}\right), z_{R}=k s_{0}^{2} / 2, E_{0}$ is the laser field amplitude, $z_{R}$ is the Rayleigh length, $s(z)$ the beam diameter at a given propagation distance, $f(\eta)=$ $\exp \left(-\eta^{2} / \tau^{2}\right), \eta=t-z / c$, and $\tau$ is the $1 / e^{2}$ temporal duration of the laser pulse.

\section{Relativistic electron dynamics}

Once the electron beam is described accurately by a sampled six-dimensional phase space distribution, the dynamics of each electron in the laser field is calculated by solving the relativistic equations of motion:

$$
\frac{d \mathbf{p}}{d t}=q\left(\mathbf{E}_{\text {laser }}+\frac{\mathbf{p}}{\gamma m} \times \mathbf{B}_{\text {laser }}\right), \quad \frac{d \mathbf{r}}{d t}=\frac{\mathbf{p}}{\gamma m},
$$

where $q$ is the charge of an electron, $\mathbf{p}$ the particle momentum, $\mathbf{E}_{\text {laser }}$ and $\mathbf{B}_{\text {laser }}$ are the laser electric and magnetic field vectors.

We use an adaptive fourth order Runge-Kutta ordinary differential equation solver [35] with relative errortolerance threshold of $10^{-6}$, a local error threshold of $10^{-12}$, and a time step typically of the order of $10^{-4}$ femtosecond. Since the electron beams considered in this paper are relativistic and have low density $\left(n_{e} / \gamma^{3} \ll 10^{16} \mathrm{~cm}^{-3}\right)$, space charge forces are neglected $[1,2]$. In the absence of the laser field, the electron beam trajectory is ballistic. Also neglected in these calculations is radiation damping since the energy radiated by an electron per passage through the laser pulse is small compared to the energy of the electron.

\section{Radiation calculation}

Once the dynamics of each electron is obtained, the energy density radiated per unit frequency $\omega$ and solid angle $\Omega$ by a single electron moving in the intense laser field can be described by the classical formula [36]

$$
\frac{d^{2} I}{d \omega d \Omega}=2|\mathbf{A}(\omega)|^{2}
$$

where

$$
\mathbf{A}(\omega)=\left(\frac{e^{2}}{8 \pi^{2} c}\right)^{1 / 2} \int_{-\infty}^{\infty} e^{i \omega t}\left[\frac{\mathbf{n} \times[(\mathbf{n}-\boldsymbol{\beta}) \times \dot{\boldsymbol{\beta}}}{(1-\boldsymbol{\beta} \cdot \mathbf{n})^{3}}\right] d t,
$$

where $e$ is the charge of an electron, $\mathbf{n}$ is a unit vector in the direction of observation, and $\boldsymbol{\beta}$ is the velocity of the electron normalized by the speed of light.

The energy radiated per unit frequency $\omega$ and solid angle $\Omega$ per electron by the electron beam, sampled with $N_{s}$ particles, is then obtained by summing over the entire phase space:

$$
\frac{d^{2} I_{\mathrm{e}}}{d \omega d \Omega}=\frac{1}{N_{s}} \sum_{i=1}^{N_{s}} \frac{d^{2} I_{i}}{d \omega d \Omega}
$$

The total energy radiated from an electron beam distribution with an integrated total electron number $N_{e}=$ $\int d^{3} \mathbf{r} d^{3} \mathbf{p} f(\mathbf{r}, \mathbf{p})$ is given by

$$
\frac{d^{2} I_{\text {total }}}{d \omega d \Omega}=N_{e} \frac{d^{2} I_{e}}{d \omega d \Omega}
$$

where $f(\mathbf{r}, \mathbf{p})$ is the electron beam phase space distribution function.

\section{BENCHMARK RESULTS AND DISCUSSION}

To benchmark our code, we used the general analytic expressions of the radiated energy density, derived by integrating the Liénard-Wiechert potentials [1,2]. Of particular interest is the limit of low strength laser fields, $a_{0}<1$, where the radiation is dominated by the first harmonic. For the results presented in this section, a laser field with intensity of $9 \times 10^{16} \mathrm{~W} / \mathrm{cm}^{2}$ corresponding to $a_{0} \approx 0.2$ is used. In this case, for small observation angle, $\theta$, the energy radiated by a single electron per unit solid angle $\Omega$ and per unit frequency $\omega$ may be written as $[37,38]$

$$
\frac{d^{2} I}{d \omega d \Omega}=r_{e} m c \gamma^{2} N_{0}^{2} a_{0}^{2}\left(\frac{\omega}{4 \gamma^{2} \omega_{0}}\right)^{2} R\left(\omega, \omega_{0}\right),
$$

where

$$
R\left(\omega, \omega_{0}\right)=\left(\frac{\sin (\bar{k} L / 2)}{\bar{k} L / 2}\right)^{2},
$$

and $k\left(k_{0}\right)$ are scattered (laser) wave numbers, $\bar{k}+k_{0}=$ $k\left(1+\gamma^{2} \theta^{2}\right) / 4 \gamma^{2}, L=N_{0} \lambda_{0}$ is the interaction length, $N_{0}$ is the number of laser periods with which the electron interacts, and $r_{e}=e^{2} / 4 \pi \epsilon_{0} m c^{2}$ is the classical electron radius.

\section{A. Electron beam with finite energy spread}

For an ultrashort electron beam with a finite energy spread and negligible divergence angle and transverse beam size, the total scattered spectrum can be estimated analytically by summing over the electron beam energy distribution, with the spectrum from a single electron given with Eq. (1). Typically, laser-wakefield accelerated electrons have large energy spread compared to the linewidth of the scattered Thomson radiation from a single electron. In this case, the resonance function $R\left(\omega, \omega_{0}\right)$ can be approximated with a delta function, and the total radiated energy per unit frequency per unit solid angle per electron may be obtained $[37,38]$ with

$$
\frac{d^{2} I_{T}}{d \omega d \Omega}=\frac{1}{2} r_{e} m c N_{0} a_{0}^{2} \gamma^{3} f(\gamma)
$$

where $f(\gamma)=\left(1 / N_{e}\right) d N / d \gamma, N_{e}$ is the total number of electrons in the beam. 
The energy distribution of an electron beam sampled with $N_{s}=10^{5}$ particles is shown in Fig. 1(a). The average energy of the beam is approximately $100 \mathrm{MeV}$ and the energy spread around the average energy is $34 \%$. Initially, the electron beam is located at $\left(x=0, y=0, z=z_{e}=\right.$ $2 c \tau)$ and travels in the negative $z$ direction. An intense plane wave laser pulse, $I_{0}=9 \times 10^{16} \mathrm{~W} / \mathrm{cm}^{2}$ and $\lambda_{0}=$ $800 \mathrm{~nm}$, located at $z=-z_{e}$ and traveling in the positive $z$ direction is backscattered by the electron beam. The resulting backscattered energy density is shown in Fig. 1(b). The radiation is calculated with an electron beam sampled with three different total numbers of electrons $\left(N_{s}=10^{4}\right.$, $3 \times 10^{4}$, and $10^{5}$ ). Overall, the radiation calculated numerically with our model is in close agreement with that obtained analytically with Eq. (2); see Fig. 1(b). The radiation spectrum calculated with the three different total number of electrons is identical with the radiation calculated with the smallest number of electrons $\left(N_{s}=10^{4}\right)$ showing small modulations in the scattered radiation. This indicates that such an electron beam sampled with $N_{s}=10^{4}$ particles provides sufficient resolution for Thomson scattering calculations. The peak radiated photon energy calculated with our model is $0.26 \mathrm{MeV}$ which compares well to the analytically obtained value of $0.27 \mathrm{MeV}$. The peak photon energy is also consistent with the formula $E_{\mathrm{sc}}=4 \gamma^{2} \hbar \omega_{0}$. The scattered number of photons per electron per unit solid angle obtained by integrating the curves shown in Fig. 1(b) is 416 photons/sr/electron.

\section{B. Scattering of a focused laser pulse by a realistic electron beam}

The two essential components of ultrashort and highenergy radiation sources that are based on the process of inverse Compton scattering are a pulsed electron accelerator and a laser undulator. Accelerators that are based on rf- and laser-plasma technologies have been proposed and employed in inverse Compton scattering experiments [37,39-41]. Electron beams generated by either of these technologies are pulsed by nature and have finite energy spread, divergence angle, and transverse beam extent. These realistic electron beam parameters must be included in inverse Compton scattering calculations to quantify the emitted radiation accurately. The transverse size of the electron beam could also be comparable to the focal spot size of the scattering laser pulse. Hence, the electron beam as a whole experiences the curved wave front of the laser even though single electrons experience local plane waves. Therefore, the plane wave approximation of the laser field is not valid. To calculate the radiation more accurately, a $\mathrm{TEM}_{00}$ spatial mode laser field with Gaussian profile is used in this subsection.

The six-dimensional phase space distribution of an electron beam that has an average energy, $E_{0}=100 \mathrm{MeV}$, and relative energy spread, $\Delta E / E_{0}=0.24$, as generated with a normal random number generator, is shown in Fig. 2. The electron beam is sampled with $10^{5}$ electrons. The electron beam which is located initially at a longitudinal position, $z_{e}=2 c \tau$, has a Gaussian temporal profile with a FWHM width of 12 femtosecond; see Fig. 2(b). On average, the electron beam travels in the negative $z$ direction. The FWHM angular spread of the electron beam is $2.4 \mathrm{mrad}$. At its initial longitudinal position, the FWHM transverse spatial width of the electron beam is $12 \mu \mathrm{m}$.

An intense Gaussian laser pulse, with $I_{0}=$ $9 \times 10^{16} \mathrm{~W} / \mathrm{cm}^{2}, \quad \lambda_{0}=800 \mathrm{~nm}$, and $s_{0}=10.2 \mu \mathrm{m}$ (corresponding to $12 \mu \mathrm{m}$ FWHM focus size), initially
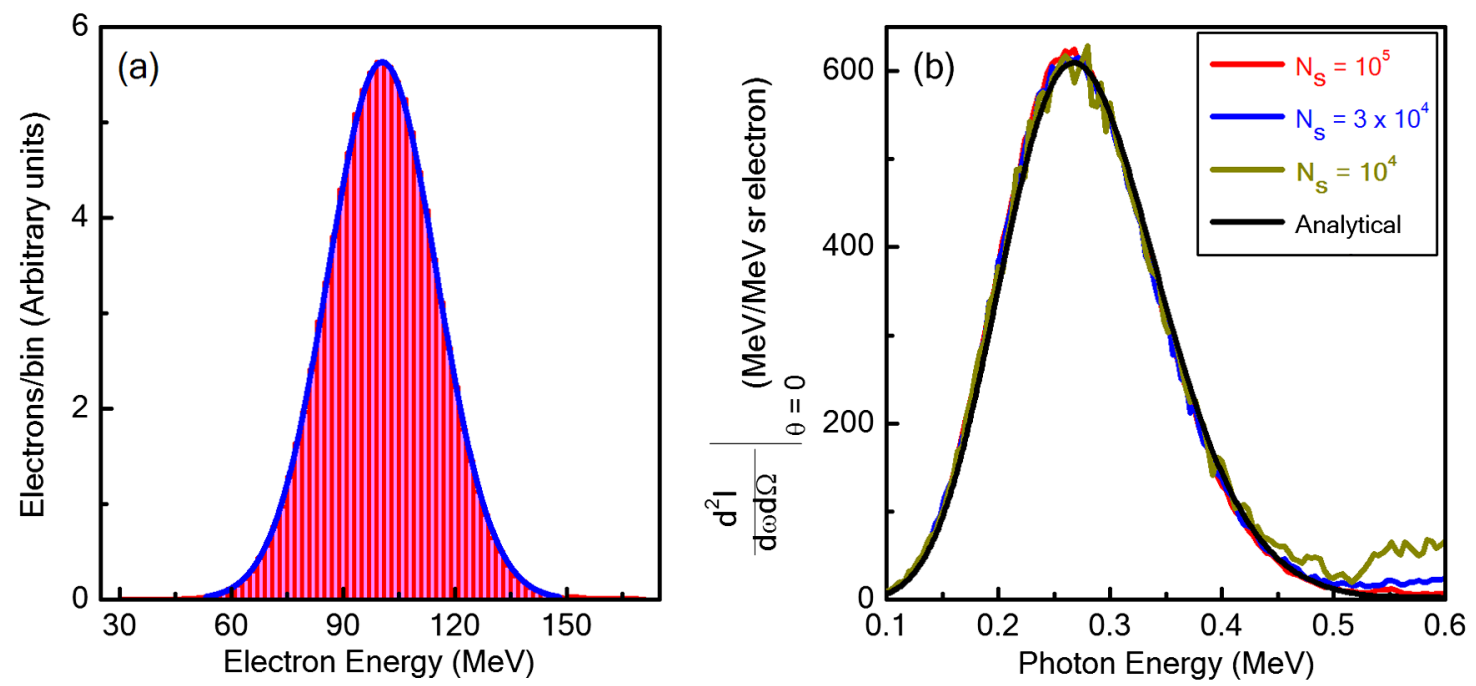

FIG. 1. Histogram of electron beam energy distribution (a), and the corresponding on-axis Thomson scattered radiated energy density (b) calculated numerically with an electron beam sampled with $N_{s}=10^{4}$ (dark green), $N_{s}=3 \times 10^{4}$ (blue), $N_{s}=10^{5}$ (red), and analytic estimate (black). The scattering laser intensity is $9 \times 10^{16} \mathrm{~W} / \mathrm{cm}^{2}, \lambda_{0}=800 \mathrm{~nm}$, and $\tau=90 \mathrm{fs}$. The energy spread of the electron beam is $34 \%$, and the corresponding Thomson bandwidth is $66 \%$. The scattered number of photons per electron per unit solid angle is 416 . 

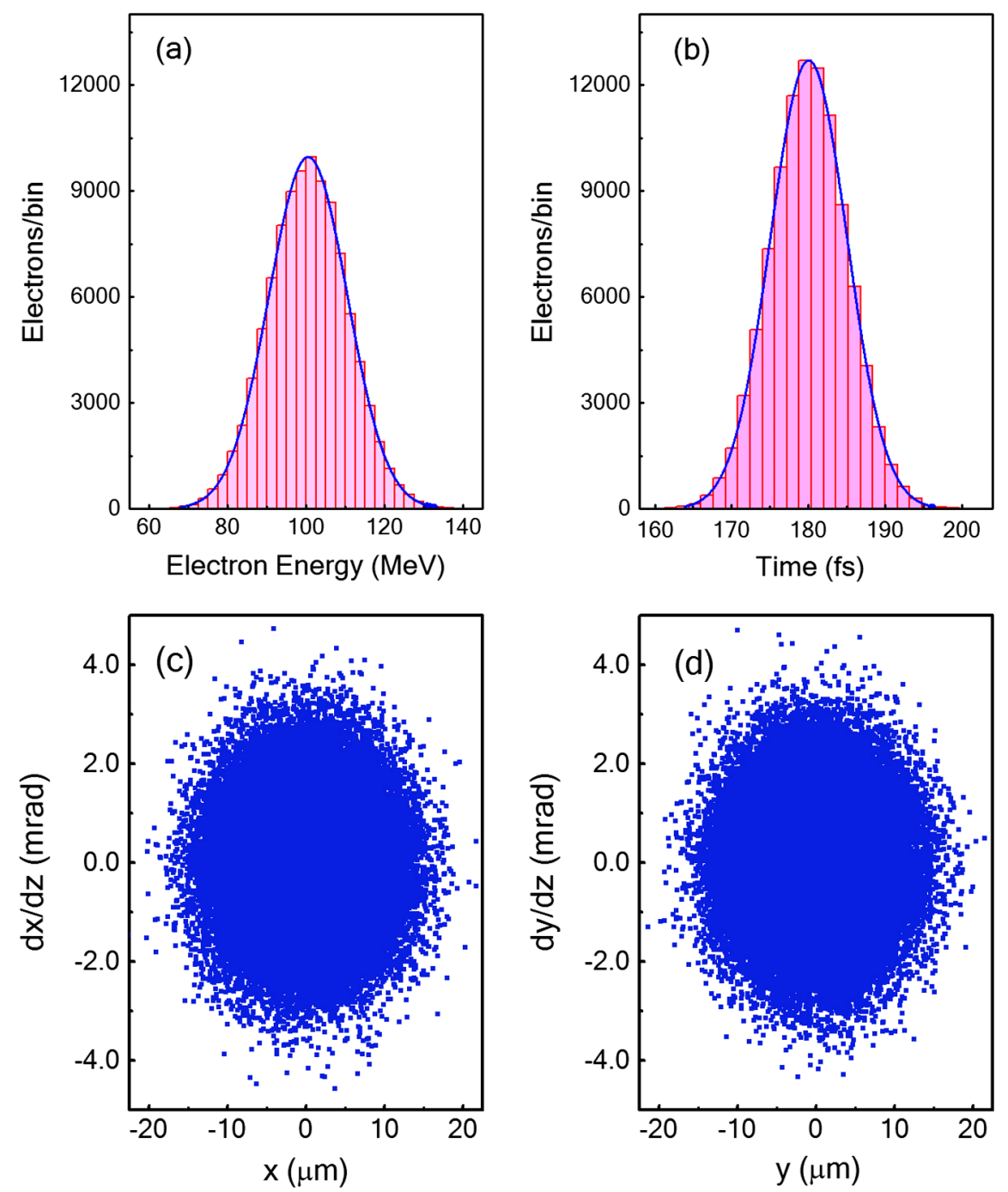

FIG. 2. Electron beam phase space, as generated by a normal random number generator. Electron beam energy spectrum (a), electron beam temporal profile (b), and transverse electron beam phase space plots (c), (d). The energy spread of the electron beam (FWHM) is $24 \%$. The electron beam angular, temporal, and spatial Gaussian profiles have corresponding FWHM widths of 2.4 mrad, 12 femtosecond, and 12 microns, respectively.

located at $z=-z_{e}$ and traveling in the positive $z$ direction is backscattered by the electron beam, and the resulting backscattered energy density is shown in Fig. 3. The backscattered energy density is calculated for four different observation polar angles $(\theta=0,1.6,3.2$, and $4.8 \mathrm{mrad})$ and in the plane parallel to the polarization direction of the scattering laser pulse. The polar angles are measured with respect to the electron beam propagation direction, i.e., the direction $\theta=0$ is parallel to the direction of electron beam propagation. Consistent with the analysis based on classical Doppler shift, the amplitude of the radiated energy density as well as the photon energy at which the energy density reaches its maximum as calculated with our model are the highest for the smallest observation angle, i.e., $\theta=0$. The calculated peak on-axis radiated photon energy is $0.23 \mathrm{MeV}$ and is consistent with the prediction by the formula $4 \gamma^{2} E_{L}$, where $E_{L}$ is the scattering laser photon energy as can be seen from Fig. 4(a). The calculated on-axis radiated energy is about $57 \mathrm{MeV} / \mathrm{sr} /$ electron and reduces to $2.1 \mathrm{MeV} / \mathrm{sr} /$ electron at an off-axis observation angle $\theta=4.8 \mathrm{mrad}$; see Fig. 4(b). Correspondingly, the calculated off-axis peak photon energy is $0.14 \mathrm{MeV}$ and is in close agreement with the analytic estimate given by $4 \gamma^{2} E_{L} /\left(1+\gamma^{2} \theta^{2}\right)$, which with $\gamma \approx 197$ predicts photon energy of $0.12 \mathrm{MeV}$.

Based on the radiation yield of a single electron moving on axis [1] and in the case of small scattering and incidence angles at nonrelativistic laser intensities, previous works $[37,42]$ provide an analytic estimate of the radiation distribution of an electron beam. The analytic estimate which assumed invariance over the azimuthal angle of the electron beam distribution involved a convolution of the form 


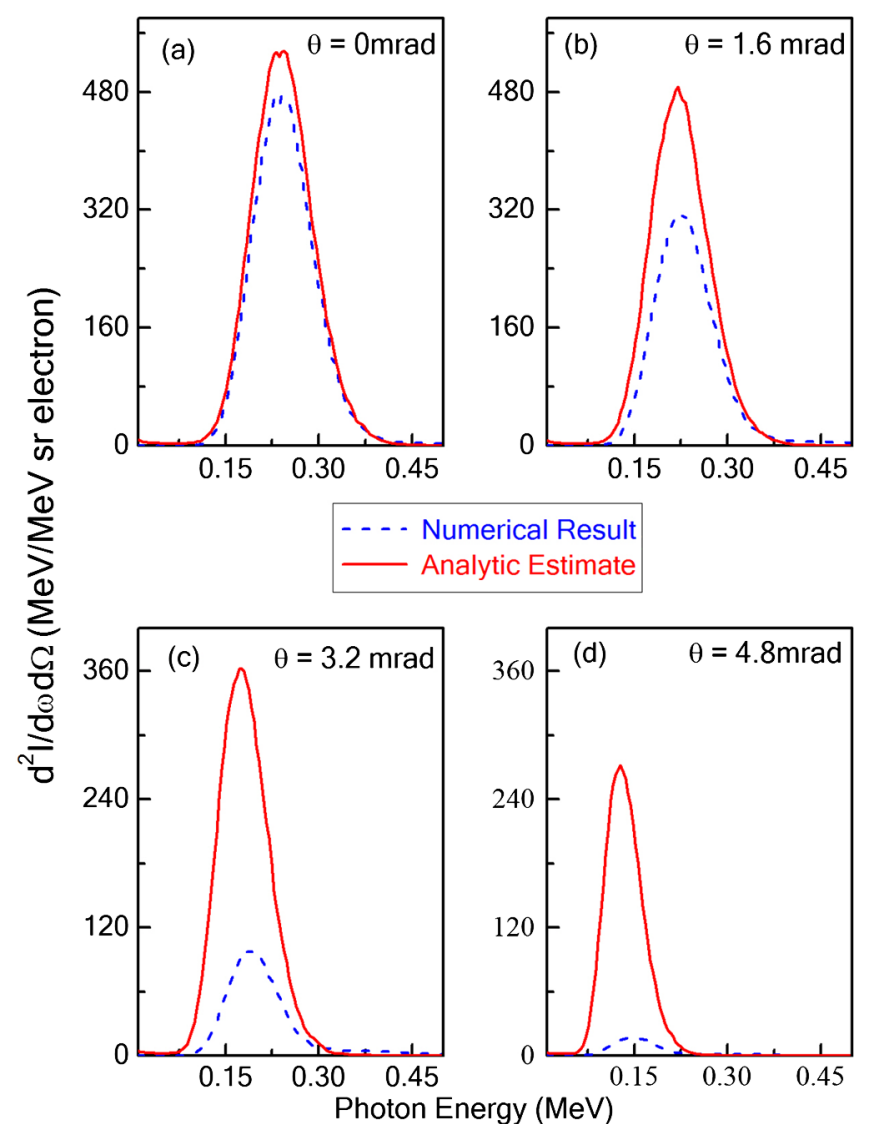

FIG. 3. Radiated energy density calculated numerically (dashed-blue line) and estimated analytically using Eq. (3) (red line). The radiation is calculated at four different observato the electron beam propagation axis and in the plane containing the polarization-propagation axis of the scattering laser pulse. For the smallest angle, $\theta$, the radiation yield calculated analytically is in a reasonable agreement with our model. The agreement gets worse as the observation angle, $\theta$, is increased.

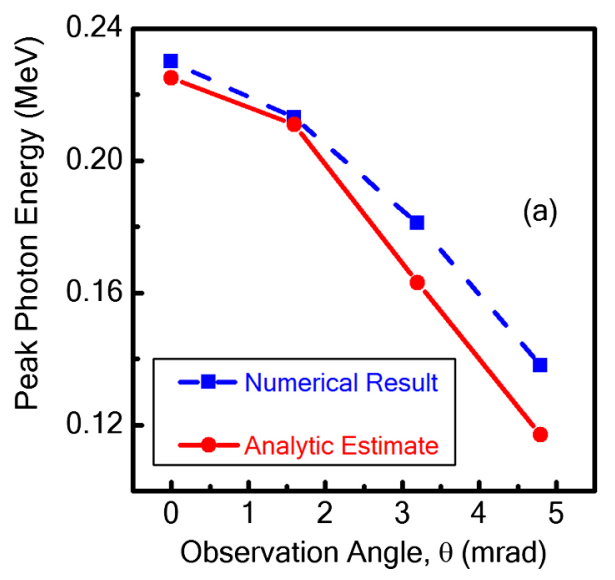
tion angles: $\theta=0,1.6,3.2$, and $4.8 \mathrm{mrad}$; measured with respect

$$
\begin{aligned}
\frac{d^{2} I_{T}}{d \omega d \Omega}= & \int d \theta_{e} d \gamma d r_{e} \exp \left(-2 r_{e}^{2} / s_{0}^{2}\right) \\
& \times f\left(\theta_{e}, \gamma\right) \frac{d^{2} I}{d \omega d \Omega}\left(\theta-\theta_{e}, \gamma, \omega\right)
\end{aligned}
$$

Equation (3) has been used to provide framework for analysis of experimental results and has proven successful in predicting on-axis radiated spectra [43]. The validity of this assumption however, needs to be quantified when using it for a full angle-and-energy resolved characterization of the scattered radiation. The scattered radiation yield obtained with Eq. (3) compared to the yield calculated using our three-dimensional model is shown in Figs. 3 and 4.

For an on-axis observation angle $(\theta=0)$, the analytic estimate based on Eq. (3) agrees reasonably well with the detailed calculation using our model. The analytic estimate is able to predict the shape of the scattered energy spectrum. This can be seen from Fig. 3(a) where the shapes of the two energy spectrum curves match quite well. Furthermore, the radiated peak photon energy obtained with our model is $0.23 \mathrm{MeV}$ which compares well with the value $0.225 \mathrm{MeV}$ obtained with Eq. (3); see Fig. 4(a). The radiated energy per unit solid angle and per electron obtained with the simplified expression given by Eq. (3) slightly overestimates the radiated energy as predicted by our model. This can be seen from Fig. 4(b) where the onaxis radiated energy density calculated with our model is $57 \mathrm{MeV} / \mathrm{sr} /$ electron compared to $64.8 \mathrm{MeV} / \mathrm{sr} /$ electron obtained with Eq. (3). The overall reasonable agreement for $(\theta=0)$ between the radiation calculated with our model and that obtained with Eq. (3) is expected since the simplified expression is based on a single electron moving on axis, i.e., $\left(\theta_{e}=0\right)$. For an on-axis observation, only electrons with $\theta_{e} \approx 0$ contribute to the radiated

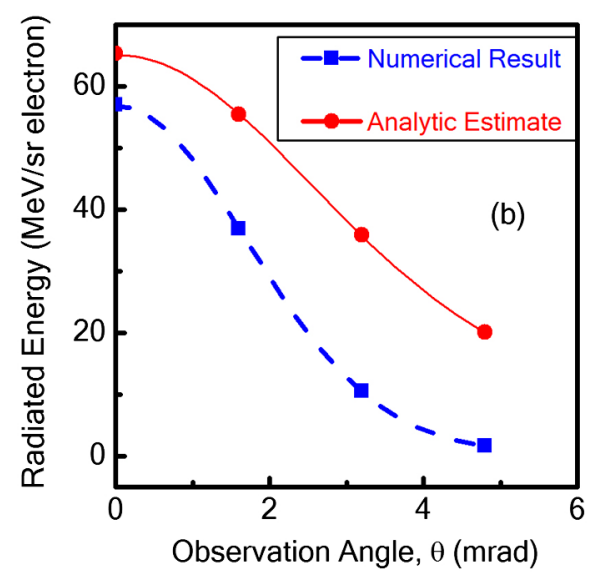

FIG. 4. Radiated peak spectral photon energy versus observation polar angle (a) calculated numerically (blue squares) and estimated analytically (red full circle). The radiated energy obtained numerically (solid blue square symbols) and estimated analytically with Eq. (3) (solid red full circle symbols) is shown in (b). The lines are to guide the eye. The photon flux obtained by integrating the curves in (a) is 0.015 photons/electron (analytic estimate) which is greater than 2 times 0.006 photons/electron obtained with our model. 
energy significantly. In this case, the azimuthal invariance assumption of the electron beam distribution is valid. This can be seen more easily with the approximate analytic expression of the radiated fundamental frequency derived in [42]. For linear scattering $\left(a_{0} \ll 1\right)$, the analytic solution that included the incident and scattering azimuthal angles may be written as $\omega_{\mathrm{sc}}=4 \gamma^{2} \omega_{0} /\left[1+(\gamma \tilde{\theta})^{2}\right]$, where $\tilde{\theta}^{2}=\theta^{2}+\theta_{e}^{2}-2 \theta \theta_{e} \cos \left(\varphi-\varphi_{e}\right)$. According to this expression, the effect of an azimuthal angle of the electron beam on the scattered photon energy is negligible for an on-axis observation angle and, hence, a reasonable agreement between our model and Eq. (3).

For an off-axis observation $(\theta=1.6 \mathrm{mrad})$, the shape of the radiated energy spectrum as well as the radiated peak photon energy obtained with Eq. (3) and calculated with our model are in reasonable agreement; see Figs. 3(b) and 4(a). The radiated energy per unit solid angle and per electron obtained with the simplified expression given by Eq. (3) is a factor of approximately 1.5 times higher than the yield calculated with the detailed computation using our three-dimensional model. This means that estimating the number of photons radiated within a collection angle of $1.6 \mathrm{mrad}$ using Eq. (3) will overestimate the radiated photon number by a factor as high as 1.5 times when compared to radiated photon number estimation using our three-dimensional model. The assumption of an azimuthal angle invariance of the electron beam distribution and radiation calculation based on an electron moving on axis implicit in the simplified expression given by Eq. (3) undermine the effect of electron beam divergence and overestimates the radiated energy.

Similarly, the agreement between the radiated energy spectrum as well as the peak photon energy calculated with our model and obtained with Eq. (3) worsens when the off-axis observation angle is increased further to $(\theta=3.2 \mathrm{mrad})$, greater than the divergence angle of the electron beam $\left(\theta_{e}=2.4 \mathrm{mrad}\right)$. This can be seen more easily from Figs. 3(c), 4(a), and 4(b). The peak photon energy obtained with Eq. (3) is $0.16 \mathrm{MeV}$ which is smaller than $0.18 \mathrm{MeV}$ computed with our model. The radiated energy per unit solid angle and per electron obtained with Eq. (3) is $36 \mathrm{MeV} / \mathrm{sr} /$ electron which is approximately a factor of 3.6 times higher than the yield obtained with our model.

The peak photon energy and the radiated energy per unit solid angle per electron calculated with our model for an observation angle $\theta=4.8 \mathrm{mrad}$ are $0.14 \mathrm{MeV}$ and $1.4 \mathrm{MeV} / \mathrm{sr} /$ electron, respectively. This can be compared to the respective values $0.12 \mathrm{MeV}$ and 20.5 MeV/sr/electron obtained with Eq. (3). The analytic estimate underestimates the radiated peak photon energy and overestimates the integrated radiated energy by an approximate factor of 15 times. Though the radiation yield from the scattering of an electron beam overestimates the radiated energy, the radiation emitted off axis from a single electron moving on axis calculated with our model is consistent with the analytic estimate [2] that is based on the plane wave approximation of the laser.

\section{SCATTERING FROM A PULSED LASER AND SPECTRAL BROADENING}

Previous investigation of the effect of beam shapes on the Thomson scattered spectrum [8-12], in particular, the pulsed nature of the laser has been shown to introduce spectral substructures within the radiated harmonics. When an electron interacts with a pulsed laser pulse, it undergoes small oscillations during the rise of the pulse, where $a(t) \ll a_{0}$, and emits radiation at the relativistic Dopplershifted laser frequency $\omega_{1}$, as shown in Fig. 5. When the laser field amplitude increases, the ponderomotive force pushes the electron backward and it emits radiation which is frequency down-shifted by a factor of $1+a_{0}^{2} / 2$. When the field decreases back to zero, the electron radiates again at $\omega_{1}$ [9]. The spectral difference in the radiation from the increasing and decreasing parts of the laser pulse results in spectral interference of the radiated field, creating oscillations in the radiated spectrum $[9,11]$. A recent study [10] has arrived at an analytic expression that relates the number of oscillations to the laser intensity and temporal duration according to the empirical formula

$$
N_{\tau}=0.24 a_{0}^{2} \tau[\mathrm{fs}]
$$

When designing a narrow-band $\gamma$-ray source for precision applications such as nuclear resonance fluorescence, this nonlinear oscillation of the spectrum must be taken into account.

This nonlinear oscillation in the spectrum may be minimized by using an appropriately chirped laser pulse. We propose a chirped laser pulse whose frequency changes with time as $\omega(t)=2 \omega_{0}\left\{1+\left[a(t) / \sqrt{2} a_{0}\right]^{2}\right\} / 3$. When the laser field amplitude increases, the radiated frequencies are down-shifted by a factor of $1+a^{2}(t) / 2$. However, for scattering with a chirped laser pulse, the frequency of the laser field is up-shifted by the same factor. This would ensure that the radiated frequencies during the period of the

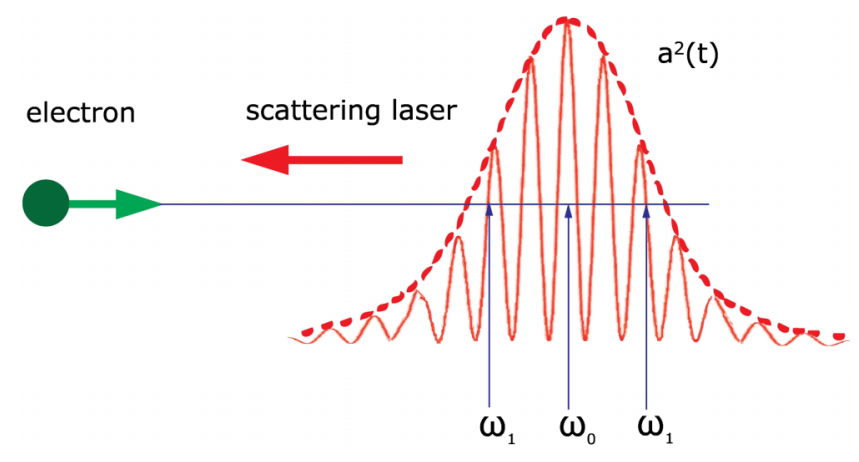

FIG. 5. Schematic diagram illustrating the time varying ponderomotive potential experienced by an electron as it collides with a counterpropagating laser pulse. 
laser pulse will be identical, and the spectral oscillations resulting from the spectral interference of the different radiated frequencies would be minimized. Since most of the radiation occurs near the peak of the laser intensity, the proposed chirp may be realized with a phase $\varphi(t)=\omega_{0} t-$ $b t^{3}+c t^{5}+\cdots$, where $b=\omega_{0} /\left(3 \tau^{2}\right)$ is a third-order phase and $c=\omega_{0} /\left(30 \tau^{4}\right)$ is a fifth order phase. For a 90 fs laser pulse, the amount of chirp required $\left(b^{-1}=\right.$ $30903 \mathrm{fs}^{3}, c^{-1}=1.08 \times 10^{8} \mathrm{fs}^{5}$ ) can be produced with conventional stretcher/compressor and pulse-shaper combinations. Figure 6 shows the radiated on-axis energy density $\left(d^{2} I / d \omega d \Omega\right)$ when an $300-\mathrm{MeV}$ electron is scattered by a pulsed laser field with temporal duration of $90 \mathrm{fs}$ FWHM and peak intensity of $1.6 \times 10^{18} \mathrm{~W} / \mathrm{cm}^{2}$. The figure shows the spectral oscillations in the radiated spectrum that are reduced in the chirped pulse case. For scattering from the transform-limited laser pulse, the radiated fundamental harmonic is split into 14 substructures which are consistent with Eq. (4). For the chirped case, the radiated spectrum is dominated by the fundamental harmonic; with only two substructures that are less prominent. This reduction of the substructures effectively reduces the bandwidth of the scattered $\gamma$-ray pulse. Integrating the curves shown in Fig. 6 gives the radiated energy per unit solid angle. The on-axis integrated photon flux per unit solid angle per electron for scattering with the chirped laser pulse is $4.1 \times 10^{4}$ photons/sr/electron, which is slightly smaller than $4.3 \times 10^{4}$ photons/sr/electron obtained for the nonchirped case. The amplitude of the radiated energy density is, however, greater by approximately 5 times for scattering with the chirped laser pulse. The bandwidth of the radiated energy density is also narrower for the chirped

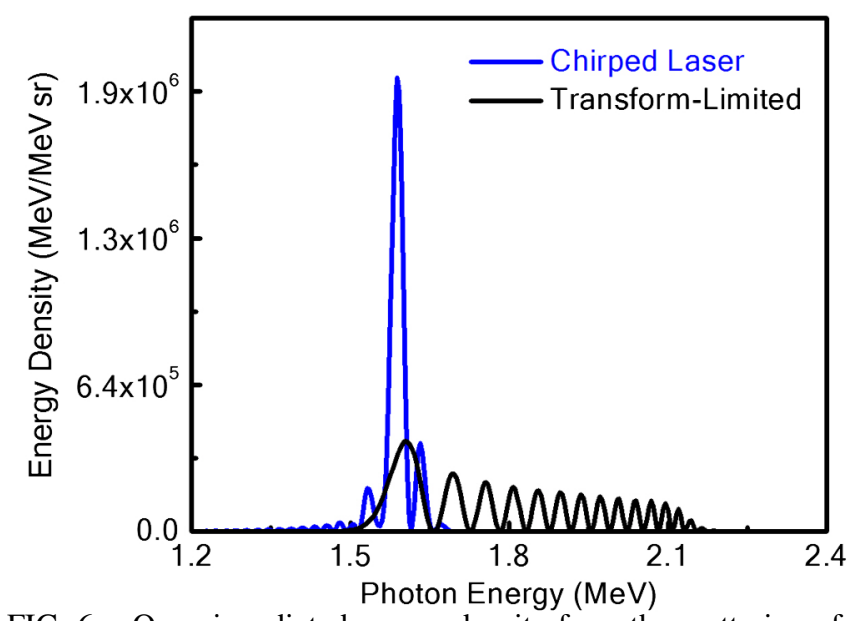

FIG. 6. On-axis radiated energy density from the scattering of a $300-\mathrm{MeV}$ electron by a $90-\mathrm{fs}$ FWHM and $1.6 \times 10^{18}-\mathrm{W} / \mathrm{cm}^{2}-$ peak-intensity chirped laser pulse (blue line) and transformlimited pulse (black line). The photon flux per unit solid angle is approximately $4.1 \times 10^{4}$ photons/sr and $4.3 \times 10^{4}$ photons $/ \mathrm{sr}$ for scattering with the chirped and transform-limited laser pulses, respectively. case. With typical electron beams from linac or LWFA based accelerators consisting of $\approx 10^{9}$ electrons, the total number of photons for this simulation (contained within a cone of half angle $1 / \gamma$ ) is $4 \times 10^{8}$. This analysis indicates that the peak on-axis brightness of the emitted radiation could be increased by approximately 5 times. Nevertheless, detailed calculation that includes realistic electron and laser beam properties as well as finite detector size is required for practical application of the chirped pulse technique in the design of narrow-band inverse Compton scattering sources. In the following subsections, we discuss the effect of finite detector size as well as realistic beam parameters on the scattered spectrum.

\section{A. Finite detector size}

In the above, the scattered radiation was calculated in a fixed direction, i.e., in the direction of the initial electron motion. But we have shown in Sec. III that the emitted radiation strongly depends on the observation angle. A finite size detector collects all photons that hit its area and, hence, measures the integrated spectrum over a certain range of observation angles. It is not clear a priori whether the observed spectral oscillations will be smeared out and if the proposed scheme of using a chirped laser reduces the bandwidth of the scattered radiation spectrum. A narrowband spectrum is desired for applications such as nuclear resonance fluorescence imaging. The smallest bandwidth of Thomson scattered radiation is limited by the linewidth of the scattering laser pulse. We chose a pinhole with a half-cone angle $\theta_{\text {cen }}=1 /(\gamma \sqrt{N})$, where $N$ is the number of optical cycles the electron interacts with, since the linewidth of the Thomson scattered radiation integrated over the solid angle subtended by the pinhole is $\propto 1 / N$ (consisting of all of the scattering laser bandwidth) and our objective is to obtain an optimized peak on-axis brightness of the Thomson radiation. The solid angle subtended by the detector should be less than the solid angle subtended by the central radiation cone, i.e., $\theta_{\text {det }}<\theta_{\text {cen }}$. Assuming an ideal detector with unity acceptance for photons hitting its surface and null acceptance otherwise, the spectrum measured by the finite size detector may be obtained by calculating the scattered radiation integrated over the solid angle subtended by the detector.

For an $300-\mathrm{MeV}$ electron interacting with a laser pulse of temporal duration FWHM $90 \mathrm{fs}$, the central radiation cone half angle is $\theta_{\text {cen }}=0.293 \mathrm{mrad}$. A circular detector with radius $R$ placed at a distance $L$ away from the interaction point along the initial electron motion subtends a collection cone of half angle $\tan \theta_{\operatorname{det}}=R / L$. The radiated energy density, collected by the detector, per unit frequency $\omega$ may then be obtained with

$$
\frac{d^{2} I_{\mathrm{det}}}{d \omega}=\sum_{j=0}^{j=K}\left(\frac{d^{2} I}{d \omega d \Omega} \times \pi\left\{[(j+1) \times \Delta \theta]^{2}-(j \Delta \theta)^{2}\right\}\right),
$$


where $K \Delta \theta=\theta_{\operatorname{det}}$. The angle subtended by the detector is divided into a total of $K$ angular bins with $\Delta \theta$ spacing between each angular bin. The spectrum should not vary significantly over the angular bin size. In the above, cylindrical symmetry of the radiation spectrum is assumed. To determine the required number of angular points for a converged result, the radiated energy density integrated over a circular detector with radius $R=1 \mathrm{~mm}$ is calculated with three different angular increments. The detector is located at a distance $D=3.4 \mathrm{~m}$ subtending a radiation collection cone of half angle $\theta_{\mathrm{det}}=0.293 \mathrm{mrad}$. The integrated energy density calculated for $K=200,500$, and 1000 corresponding to $\Delta \theta=1.47,0.587$, and $0.293 \mu \mathrm{rad}$ angular steps, respectively is shown in Fig. 7. The figure shows at least 500 angular bins are required for a converged result. Integrating the curve shown in Fig. 7 $(K=1000)$ and dividing the result by the peak photon energy $(\approx 1.6 \mathrm{MeV})$ gives $4 \times 10^{-3}$ photons/electron collected by the detector $\left(\theta_{\text {det }}=0.293 \mathrm{mrad}\right)$. With typical electron beams consisting of $\approx 10^{9}$ electrons, the total number of $x$-ray flux collected by the detector for this simulation is $4 \times 10^{6}$ photons.

To the best of our knowledge, the harmonic substructures shown in Fig. 7 have never been observed experimentally. This may be due to the small spectral separation among neighboring subpeaks $(<5 \%)$ which may easily get smeared out due to realistic electron and laser beam parameters. Previous study [10] indicates that the experimental observation of the subpeaks (without getting smeared out) shown in Fig. 7 may not be possible for scattering with the laser and electron beam parameters used in this study.

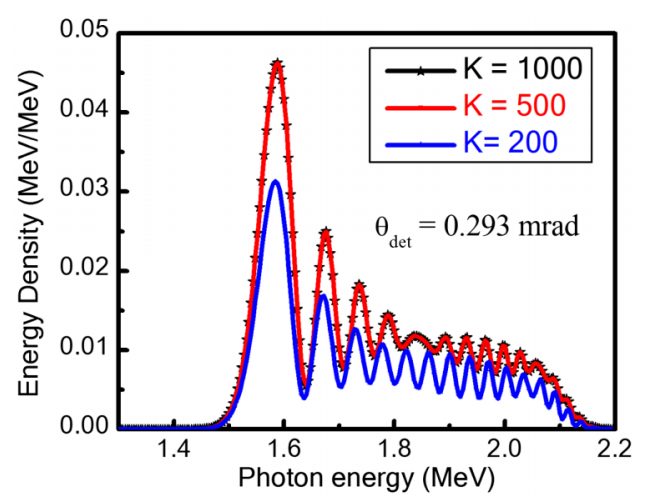

FIG. 7. Radiated energy density from the scattering of a $300-\mathrm{MeV}$ electron by a $90-\mathrm{fs}$ FWHM and $1.6 \times 10^{18}-\mathrm{W} / \mathrm{cm}^{2}-$ peak-intensity transform-limited pulse integrated over a detector subtending a cone of half angle $\theta_{\text {det }}=0.293 \mathrm{mrad}$. The angle subtended by the detector was divided into 200 (blue line), 500 (red line), and 1000 (black line) differential angular bins. The spectral intensity at each angular bin was calculated and the radiation density was then integrated over the detector. Integrating the curve shown $(K=1000)$ and dividing the result by the peak photon energy $(\approx 1.6 \mathrm{MeV})$ gives $4 \times 10^{-3}$ photons/electron collected by the detector $\left(\theta_{\text {det }}=0.293 \mathrm{mrad}\right)$.
Though the substructures are smeared out with realistic beam parameters, they broaden the bandwidth of the scattered radiation spectrum. In this paper we concentrate on the spectral broadening in the scattered radiation spectrum due to the pulsed nature of laser and discuss how it can be minimized.

Figure 8 shows the radiated energy density from scattering a $300-\mathrm{MeV}$ electron by a 90 -fs FWHM and $1.6 \times 10^{18}-\mathrm{W} / \mathrm{cm}^{2}$-peak-intensity chirped laser pulse and by a transform-limited pulse (solid black line), integrated over three different detectors subtending a radiation collection cone of half angles $\theta_{\operatorname{det}}=73.25 \mu \mathrm{rad}$, $\theta_{\mathrm{det}}=146.5 \mu \mathrm{rad}$, and $\theta_{\mathrm{det}}=293 \mu \mathrm{rad}$. As expected, a larger detector collects more photons. For scattering with the transform-limited laser pulse, the contrast of the subharmonic structures is identical when the energy density is integrated with the two smaller detectors $\left(\theta_{\text {det }}=73.25 \mu \mathrm{rad}, \theta_{\text {det }}=146.5 \mu \mathrm{rad}\right)$. However, the energy density integrated over the largest detector $\left(\theta_{\mathrm{det}}=293 \mu \mathrm{rad}\right)$ shows a reduced contrast of the subharmonic peaks. The radiated energy density collected by the three different detector sizes has the narrowest bandwidth and the highest amplitude in the case of scattering from the chirped laser pulse compared to scattering from the transform-limited laser pulse. The total number of photons $\left(N_{\gamma}\right)$ collected by the three detectors from the scattering of an $300-\mathrm{MeV}$ electron beam with $10^{9}$ electrons may be obtained by integrating the curves shown in Fig. 8 and multiplying the result by the number of electrons. For this simulation, the number of photons scattered by a transform-limited and chirped laser pulse is approximately equal. With such an electron beam, $3.3 \times 10^{5}, 8.1 \times 10^{5}$, and $4 \times 10^{6}$ photons are collected by the detectors with $\theta_{\mathrm{det}}=73.25 \mu \mathrm{rad}, \theta_{\mathrm{det}}=146.5 \mu \mathrm{rad}$, and $\theta_{\mathrm{det}}=$ $293 \mu \mathrm{rad}$, respectively.

\section{B. Practical considerations of the pulse chirping technique}

The analysis of emitted radiation from the scattering of a single electron by a plane wave laser pulse is an idealization and has to be checked when using the concept of a chirped laser pulse in the design of a narrow-band inverse Compton scattering source. A realistic laser pulse at focus has curved wave fronts with spatially dependent field amplitude. To leading order, the effect of wave front curvature on the radiated spectrum can be estimated by $\Delta \omega_{\mathrm{sc}} / \omega_{\mathrm{sc}} \approx 0.25 \times\left(\lambda_{0} / \pi s_{0}\right)^{2}$, where $s_{0}$ is the $1 / e^{2}$ intensity radius of the laser pulse [33]. For an 800-nm laser pulse focused to a spot size $s_{0}=14 \mu \mathrm{m}$, the broadening due to wave front curvature is $\Delta \omega_{\mathrm{sc}} / \omega_{\mathrm{sc}} \approx 8 \times 10^{-5}$ and is much less than the broadening due to the pulsed nature of a 90 -fs laser that is $1 / N=0.03$. Though the effect of wave front curvature is small and can be neglected, the spatial variation of the laser field amplitude can have a significant effect on the scattered spectrum since the electron beam 

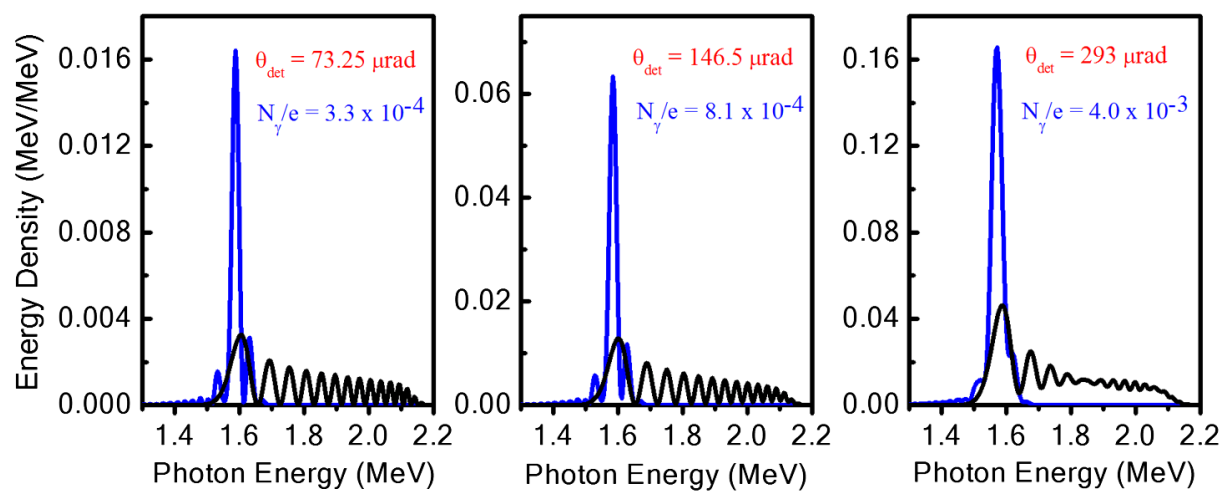

FIG. 8. Energy density integrated over a finite detector size from the scattering of a 300-MeV electron by a 90-fs FWHM and $1.6 \times 10^{18}-\mathrm{W} / \mathrm{cm}^{2}$-peak-intensity chirped laser pulse (blue line) and transform-limited pulse (solid black line). The energy density is calculated for three different detectors subtending a collection cone with half angles: $\theta_{\text {det }}=73.25 \mu$ rad (left panel), $\theta_{\text {det }}=146.5 \mu \mathrm{rad}$ (center panel), and $\theta_{\text {det }}=293 \mu \mathrm{rad}$ (right panel). The number of photons per electron $\left(N_{\gamma} / \mathrm{e}\right)$ collected by a detector for the chirped laser pulse (blue line) is shown in the figure.

transverse size could be comparable to the laser beam waist. Therefore, the plane wave approximation of the laser field may not be valid and the focused nature of the laser field has to be included when calculating the scattered radiation. Moreover, electron beams obtained with rf and laser-plasma technologies have finite energy spread and emittance. Hence, these electron and laser beam properties must be included in the calculation of the scattered spectrum when designing a narrow-band inverse Compton scattering source based on a chirped pulse.

High quality electron beams with normalized emittance of $0.35 \mathrm{~mm}$ mrad at $163 \mathrm{MeV}$ with $0.16 \%$ energy spread and a $7.24 \mu \mathrm{m}$ root-mean-square focus size could be generated with an $x$-band linac [44]. On the other hand, based on a laser-plasma accelerator technology, the production of electron beams with an average energy of $86 \mathrm{MeV}$, energy spread 2\%, and angular divergence of $3 \mathrm{mrad}$ (FWHM) have been demonstrated in laser-wakefield acceleration
(LWFA) experiments [45]. It has been shown that LWFA electron beams have a source size on the order of a few $\mu \mathrm{m}$. The calculated on-axis energy density from scattering of a 90 -fs FWHM and $1.6 \times 10^{18}-\mathrm{W} / \mathrm{cm}^{2}$-peak-intensity chirped laser pulse and transform-limited pulse using both the $x$-band linac and LWFA electron beams is shown in Fig. 9. We considered two models for the laser pulse, namely, plane wave and a focused (14 $\mu \mathrm{m}$ FWHM) $\mathrm{TEM}_{00}$ mode with Gaussian spatial profile. As can be seen from Fig. 9(a), the spectral oscillations in the radiated spectrum are well resolved in the case of scattering with a transform-limited plane wave laser pulse using the $x$-band linac. In the case of scattering from a chirped plane wave laser pulse using the $x$-band linac, the spectral oscillations are reduced significantly and the radiated energy density shows a single peak with amplitude 5 times greater than the scattered amplitude using a transform-limited plane wave laser pulse. The scattered energy density using an $x$-band
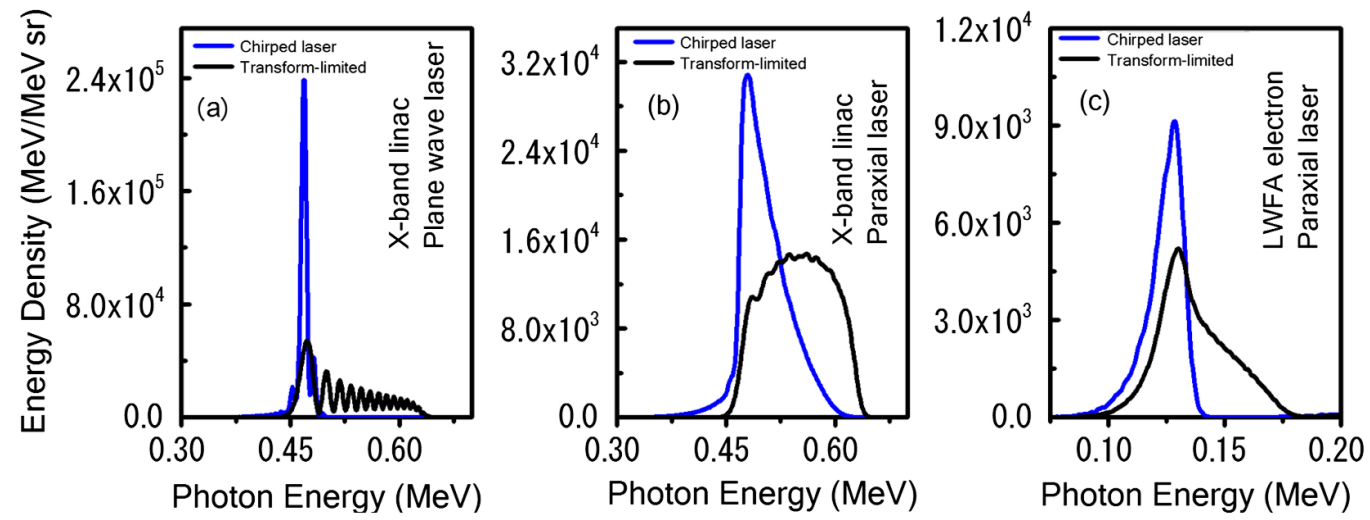

FIG. 9. Scattered on-axis energy density per electron from the interaction of an $x$-band linac electron beam with a 90-fs FWHM and $1.6 \times 10^{18}-\mathrm{W} / \mathrm{cm}^{2}$-peak-intensity transform-limited (black line) and chirped laser (blue line) under the plane wave approximation (a) and a TEM $_{00}$ spatial mode with Gaussian profile (b). The scattered on-axis energy density from a LWFA electron beam using a Gaussian transform-limited laser focus (black line) and chirped laser focus is shown in (c). The FWHM beam sizes of the laser focus and the LWFA electron beam are 14 and $2 \mu \mathrm{m}$, respectively. 
linac with transform-limited and chirped paraxial laser focus is shown in Figs. 9(b) and 9(c). The figures show the spectral oscillations in the radiated spectrum (observed with transform-limited laser pulse) are smeared out for scattering in a realistic transform-limited laser focus due to the large $x$-band linac electron beam size and large energy spread LWFA electrons. The bandwidth (amplitude) of the scattered energy density in the chirped laser case using the $x$-band linac is 0.38 (1.82) times that of the transform-limited laser focus. Thus, the peak on-axis spectral brightness could be increased by a factor of 5 times.

The scattered energy density using the LWFA electron beam and realistic laser focus is shown in Fig. 9(c). The energy density calculated with the plane wave laser (not shown here) is identical with the focused laser case since the LWFA electron beam has small focal size. For LWFA electron beams, spectral oscillations are smeared out due to the large electron energy spread. However, the peak on-axis brightness of the scattered radiation can still be increased by scattering from a chirped laser pulse. The bandwidth (amplitude) of the scattered energy density using the chirped laser pulse is 0.5 (1.75) times that of the transform-limited laser focus using the LWFA electron beam. Thus, the peak on-axis spectral brightness could be increased by about 4 times.

For both the $x$-band linac and LWFA electron beams, the divergence of the electron beams $\theta_{e}$ is smaller than the full radiation cone with a half angle of $1 / \gamma$, the photon flux contained within a cone of half angle $\theta_{e}$ may be obtained by integrating the on-axis spectral intensity shown in Fig. 9. The scattered number of photons per electron is 0.01 photons/electron, and 0.04 photons/electron for scattering of the $x$-band linac and LWFA electrons by a paraxial laser focus, respectively.

\section{CONCLUSION}

In summary, based on single-particle-trajectory tracking, a relativistic three-dimensional nonlinear Thomson scattering code has been developed and benchmarked against analytical expressions for scattering in a plane wave laser field. It is shown that the use of a simplified analytic expression that is based on the radiation yield of a single electron moving on-axis overestimates the total radiation yield from the scattering of a laser focus by a relativistic electron beam and underestimates the radiated peak photon energy.

Scattering from a pulsed laser results in nonlinear spectral modulation in the radiated spectrum. These modulations broaden the radiated spectrum and should be taken into account when designing narrow-band radiation sources that are based on the process of inverse Compton scattering. By using an appropriately chirped laser pulse, we show that the oscillations in the radiated spectrum can be minimized significantly. The required amount of chirp can be produced with conventional stretcher/compressor and pulse-shaper combinations. This concept might be used in the design of narrow-band $\gamma$-ray sources that are based on scattering from relativistic electron beams generated both with rf- and laser-plasma based accelerator technologies.

\section{ACKNOWLEDGMENTS}

We would like to acknowledge useful discussions with S. Y. Kalmykov and S. Banerjee. This work was completed utilizing the Holland Computing Center of the University of Nebraska. This work was supported by the U.S. Department of Energy: No. DE-FG02-05ER15663 and No. DE-FG02-08ER55000; Defense Threat Reduction Agency: No. HDTRA1-11-C-0001; Air Force Office for Scientific Research: No. FA 9550-08-1-0232 and No. FA9550-11-1-0157; Department of Homeland Security: No. 2007-DN-007-ER0007-02; and Defense Advanced Research Projects Agency: No. FA9550-09-10009 and No. FA9550-07-1-0521.

[1] E. Esarey, S. K. Ride, and P. Sprangle, Phys. Rev. E 48, 3003 (1993).

[2] S. K. Ride, E. Esarey, and M. Baine, Phys. Rev. E 52, 5425 (1995).

[3] F. Albert, S. G. Anderson, D. J. Gibson, C. A. Hagmann, M. S. Johnson, M. Messerly, V. Semenov, M. Y. Shverdin, B. Rusnak, A. M. Tremaine, F. V. Hartemann, C.W. Siders, D. P. McNabb, and C.P. J. Barty, Phys. Rev. ST Accel. Beams 13, 070704 (2010).

[4] E. S. Sarachik and G. T. Schappert, Phys. Rev. D 1, 2738 (1970).

[5] M. Babzien, I. Ben-Zvi, K. Kusche, I. Pavlishin, I. Pogorelsky, D. Siddons, V. Yakimenko, D. Cline, F. Zhou, T. Hirose, Y. Kamiya, T. Kumita, T. Omori, J. Urakawa, and K. Yokoya, Phys. Rev. Lett. 96, 054802 (2006).

[6] T. Kumita, Y. Kamiya, M. Babzien, I. Ben-Zvi, K. Kusche, I. V. Pavlishin, I. V. Pogorelsky, D. P. Siddons, V. Yakimenko, T. Hirose, T. Omori, J. Urakawa, K. Yokoya, D. Cline, and F. Zhou, Laser Phys. 16, 267 (2006).

[7] C. Bula, K. McDonald, E. Prebys, C. Bamber, S. Boege, T. Kotseroglou, A. Melissinos, D. Meyerhofer, W. Ragg, D. Burke, R. Field, G. HortonSmith, A. Odian, J. Spencer, D. Walz, S. Berridge, W. Bugg, K. Shmakov, and A. Weidemann, Phys. Rev. Lett. 76, 3116 (1996).

[8] F. V. Hartemann, F. Albert, C. W. Siders, and C. P. J. Barty, Phys. Rev. Lett. 105, 130801 (2010).

[9] P. Michel, E. Esarey, C. B. Schroeder, C. Tóth, W.P. Leemans, and D. Schneider, AIP Conf. Proc. 737, 393 (2004).

[10] T. Heinzl, D. Seipt, and B. Kaempfer, Phys. Rev. A 81, 022125 (2010).

[11] C. Brau, Phys. Rev. ST Accel. Beams 7, 020701 (2004).

[12] G. A. Krafft, Phys. Rev. Lett. 92, 204802 (2004). 
[13] F. He, Y. Y. Lau, D. P. Umstadter, and R. Kowalczyk, Phys. Rev. Lett. 90, 055002 (2003).

[14] K. Kawase, M. Kando, T. Hayakawa, I. Daito, S. Kondo, T. Homma, T. Kameshima, H. Kotaki, L.-M. Chen, Y. Fukuda, A. Faenov, T. Shizuma, M. Fujiwara, S. V. Bulanov, T. Kimura, and T. Tajima, Rev. Sci. Instrum. 79, 053302 (2008).

[15] W. Bertozzi, J.A. Caggiano, W. K. Hensley, M. S. Johnson, S. E. Korbly, R. J. Ledoux, D. P. McNabb, E. B. Norman, W. H. Park, and G. A. Warren, Phys. Rev. C 78, 041601 (2008).

[16] D. Sober et al., Nucl. Instrum. Methods Phys. Res., Sect. A 440, 263 (2000).

[17] K. Hirose et al. Nucl. Instrum. Methods Phys. Res., Sect. A 564, 100 (2006).

[18] L. I. Schiff, Phys. Rev. 83, 252 (1951).

[19] K. Kawase, Y. Arimoto, M. Fujiwara, S. Okajima, M. Shoji, S. Suzuki, K. Tamura, T. Yorita, and H. Ohkuma, Nucl. Instrum. Methods Phys. Res., Sect. A 592, 154 (2008).

[20] I. Sakai, T. Aoki, K. Dobashi, M. Fukuda, A. Higurashi, T. Hirose, T. Iimura, Y. Kurihara, T. Okugi, T. Omori, J. Urakawa, M. Washio, and K. Yokoyo, Phys. Rev. ST Accel. Beams 6, 091001 (2003).

[21] R. Schoenlein, W. Leemans, A. Chin, P. Volfbeyn, T. Glover, P. Balling, M. Zolotorev, K. Kim, S. Chattopadhyay, and C. Shank, Science 274, 236 (1996).

[22] W. Leemans, R. Schoenlein, P. Volfbeyn, A. Chin, T. Glover, P. Balling, M. Zolotorev, K. Kim, S. Chattopadhyay, and C. Shank, Phys. Rev. Lett. 77, 4182 (1996).

[23] I. Hsu, C. Chu, and C. Yu, Phys. Rev. E 54, 5657 (1996).

[24] M. Baylac, E. Burtin, C. Cavata, S. Escoffier, B. Frois, D. Lhuillier, F. Marie, J. Martino, D. Neyret, T. Pussieux, P. Bertin, C. de Jager, and J. Mitchell, Phys. Lett. B 539, 8 (2002).

[25] T. Omori, M. Fukuda, T. Hirose, Y. Kurihara, R. Kuroda, M. Nomura, A. Ohashi, T. Okugi, K. Sakaue, T. Saito, J. Urakawa, M. Washio, and I. Yamazaki, Phys. Rev. Lett. 96, 114801 (2006).

[26] R. Hajima, N. Kikuzawa, N. Nishimori, T. Hayakawa, T. Shizuma, K. Kawase, M. Kando, E. Minehara, H. Toyokawa, and H. Ohgaki, Nucl. Instrum. Methods Phys. Res., Sect. A 608, S57 (2009).

[27] N. Kikuzawa, R. Hajima, N. Nishimori, E. Minehara, T. Hayakawa, T. Shizuma, H. Toyokawa, and H. Ohgaki, Appl. Phys. Express 2, 036502 (2009).

[28] T. Hayakawa, H. Ohgaki, T. Shizuma, R. Hajima, N. Kikuzawa, E. Minehara, T. Kii, and H. Toyokawa, Rev. Sci. Instrum. 80, 045110 (2009).
[29] W. J. Brown, S. G. Anderson, C. P. J. Barty, S. M. Betts, R. Booth, J. K. Crane, R. R. Cross, D. N. Fittinghoff, D. J. Gibson, F. V. Hartemann, E. P. Hartouni, J. Kuba, G. P. Le Sage, D. R. Slaughter, A. M. Tremaine, A. J. Wootton, P. T. Springer, and J.B. Rosenzweig, Phys. Rev. ST Accel. Beams 7, 060702 (2004).

[30] I. Ghebregziabher and B.C. Walker, Phys. Rev. A 76, 023415 (2007).

[31] I. Ghebregziabher and B. C. Walker, Phys. Rev. A 77, 023417 (2008).

[32] I. Ghebregziabher, B. Shadwick, and D. Umstadter, arXiv:1204.1068.

[33] W. J. Brown and F. V. Hartemann, Phys. Rev. ST Accel. Beams 7, 060703 (2004).

[34] F. Hartemann, D. Gibson, W. Brown, A. Rousse, K. Phuoc, V. Mallka, J. Faure, and A. Pukhov, Phys. Rev. ST Accel. Beams 10, 011301 (2007).

[35] R.W. Brankin, I. Gladwell, and L.F. Shampine, RKSUITE: a Suite of Runge-Kutta Codes for the Initial Value Problem for ODEs (Department of Mathematics, Southern Methodist University, Dallas, TX, 1992), Softreport 92-S1.

[36] J.D. Jackson, Classical Electrodynamics (Wiley, New York, 1975), p. 654.

[37] P. Catravas, E. Esarey, and W. P. Leemans, Meas. Sci. Technol. 12, 1828 (2001).

[38] H. Schwoerer, B. Liesfeld, H.P. Schlenvoigt, K. U. Amthor, and R. Sauerbrey, Phys. Rev. Lett. 96, 014802 (2006).

[39] S. G. Anderson, C. P. J. Barty, S. M. Betts, W. J. Brown, J. K. Crane, R. R. Cross, D. N. Fittinghoff, D. J. Gibson, F. V. Hartemann, J. Kuba, G. P. LeSage, J. B. Rosenzweig, D. R. Slaughter, P. T. Springer, and A. M. Tremaine, Appl. Phys. B 78, 891 (2004).

[40] D. Oulianov, R. Crowell, D. Gosztola, and Y. Li, Nucl. Instrum. Methods Phys. Res., Sect. B 241, 82 (2005).

[41] P. Sprangle, A. Ting, E. Esarey, and A. Fisher, J. Appl. Phys. 72, 5032 (1992).

[42] P. Tomassini, A. Giulietti, D. Giulietti, and L. A. Gizzi, Appl. Phys. B 80, 419 (2005).

[43] Y. Mori, H. Kuwabara, K. Ishii, R. Hanayama, T. Kawashima, and Y. Kitagawa, Appl. Phys. Express 5, 056401 (2012).

[44] F. Albert, F. V. Hartemann, S. G. Anderson, R. R. Cross, D. J. Gibson, J. Hall, R. A. Marsh, M. Messerly, S. S. Wu, C. W. Siders, and C. P. J. Barty, Phys. Plasmas 19, 059902 (2012).

[45] C. G. R. Geddes, C. Toth, J. van Tilborg, E. Esarey, C. B. Schroeder, D. Bruhwiler, C. Nieter, J. Cary, and W.P. Leemans, Nature (London) 431, 538 (2004). 